\title{
Selenium deficiency and brain functions: the significance for methylmercury toxicity
}

\author{
Chiho WATANABE \\ Department of Human Ecology, Graduate School of Medicine, University of Tokyo, Tokyo
}

\begin{abstract}
Selenium has been long recognized as one of the essential trace elements. Although many selenoproteins have been identified in the last decade, the physiological roles of Se and selenoproteins remain to be elucidated. Since iodothyronine deiodinases (DIs), which regulate the tissue levels of thyroid hormone, are (likely to be) selenoproteins, Se might have specific roles for developing brain. In fact, when rodents are depleted of Se perinatally, the thyroid hormone economy of the fetus is disturbed, which may lead to the abnormal development of the brain and to the abnormal postnatal behavior observed in Se-deficient animals. When the animals were depleted of Se after weaning, when the role of thyroid hormone on brain development is minimal, neurochemical and neurophysiological alterations were found in the dopaminergic system. These postnatally-depleted rodents also showed abnormal open-field behavior, which was distinct from that observed with perinatally-depleted animals. The molecular events that convert Se-deficient status to these neurochemical, neurophysiological, and behavioral functions are largely unknown, and need to be further examined.

The interaction between Se and mercury compounds has also been the focus of many research, but there have been few reports on the interaction between the physiological (nutritional) level of Se and the toxicity of prenatal methylmercury $(\mathrm{MeHg})$. Experimental findings showed that Se-deficient rodents are more susceptible to the prenatal toxicity of $\mathrm{MeHg}$. It is noteworthy that $\mathrm{MeHg}$ specifically altered the metabolism of Se in fetal/neonatal brain. Significance of the alteration of the activities of selenoenzymes such as glutathione peroxidase and DIs in animals by prenatal $\mathrm{MeHg}$ exposure are discussed in relation to the neurobehavioral toxicity of $\mathrm{MeHg}$.
\end{abstract}

Jpn J Hyg 2001; 55: 581-9

\section{Special Categories of Elderly Patients with Intractable Neurological Diseases (NANBYO) in Toyama, Japan}

\author{
Kinuko OMORI and Teruhiko KIDO \\ School of Health Sciences, Faculty of Medicine, Kanazawa University, Kanazawa
}

\begin{abstract}
Summary The purpose of this study was to elucidate special categories of elderly patients with intractable neurological diseases (NANBYO) in Toyama, Japan. This goal was accomplished through comparisons of data gathered in 1991 and 1997. Forty-eight (20 males, 28 females) with such diseases were recruited for the study and agreed to be interviewed. The surveys were performed by three public health center nurses. The same questionnaire was used in both years. The surveys show that the number of jobless patients and those who needed total support increased by approximately $20 \%$ from 1991 to 1997 . In addition, it appears that the ratio of hospitalized patients and those who needed medical equipment installed at home increased considerably. The number of patients with caregivers increased by approximately $30 \%$. As the caregivers got older, it was observed that the patients became more anxious about their health condition. Also, in both years, both patients and caregivers showed the highest concern about a sudden change in the patients' conditions. In conclusion, an assessment of patient and family needs is important for the development of a community health care system. It is also essential for all the parties in the field of health, medicine, and welfare, as well as volunteer groups, to have a good understanding of each other's services and to have a cooperative relationship coordinated by health centers through discussion of each individual case.
\end{abstract}




\title{
Structural Analysis for Psychosocial Factors Including Health Locus of Control (HLC) and Sense of Coherence (SOC) Associated with Lifestyle-related Diseases
}

\author{
Yukie OGAWA*1, Hiroyuki NAKAMURA*2, Hirofumi NAGASE*2, \\ Keiki OGINO*2, Yoshiko OOSHITA*3 and Setsuko TSUKAHARA*1 \\ *' Faculty of Medicine School of Nursing, Toyama Medical and Pharmaceutical University, Toyama \\ *2 Department of Public Health, Kanazawa University School of Medicine, Kanazawa \\ ${ }^{*}$ Department of Food Science and Nutrition, Nishikyushu University, Saga
}

\begin{abstract}
The purpose of this study was to clarify psychosocial characteristics associated with preventive health behavior for lifestyle-related diseases. The author performed objective health examinations and gave questionnaires to 289 men (39.7 \pm 11.8 years, mean $\pm S D)$ and 80 women (32.8 \pm 10.4 years) engaged in office work. Psychosocial factors included lifestyle and perceived stress, as well as the health locus of control (HLC) and sense of coherence (SOC) as newly developed indicators for health behavior. The principal component analysis for men did not extract lifestyle from the psychosocial structures. Multiple regression analysis showed that internal HLC (IHLC), chance HLC (CHLC), professional HLC (PHLC) and stress significantly contributed to SOC. Principal component analysis using psychosocial factors in women showed two psychosocial structures, i. e. the second principal (high SOC, high lifestyle, and low stress) and the 4th principal components (high supernatural HLC, and high PHLC). Both components were negatively correlated with systolic blood pressure. SOC was recognized to be negatively associated with age, stress, and total cholesterol, and positively with IHLC, FHLC, lifestyle, and $\gamma$-GTP using multiple regression analysis for women. These results indicated a distinguishable sex difference regarding the involvement of psychosocial factors including HLC and SOC in objective health. SOC seems likely to be involved not in objective health, but closely with stress, suggesting a direct influence on mental health. Lifestyle should be divided into more detailed categories such as smoking and salt intake. Structural analysis of women suggests that SOC is involved directly or indirectly through lifestyle in objective health, different from men. To further clarify causal relationships between psychosocial factors and risk factors for lifestyle-related diseases, a longitudinal study is necessary based on these results.
\end{abstract}

Jpn J Hyg 2001; 55: 597-606

\section{Effects of Ethanol on the Nervous and Vascular Systems: -The Mechanisms of Alcohol-Induced Hypertension-}

\author{
Ichiro WAKABAYASHI*1 and Katsuhiko HATAKE*2 \\ *' Department of Hygiene \& Preventive Medicine, School of Medicine, Yamagata University, Yamagata \\ *2Department of Legal Medicine, Nara Medical University, Nara
}

\begin{abstract}
Many previous experimental and epidemiological studies have shown that alcohol consumption has a positive correlation with the incidence of hypertension. The effects of ethanol on the nervous and vascular systems in relation to the mechanisms of alcohol-induced hypertension proposed so far are reviewed here. Alcohol ingestion influences many pathophysiological functions which regulate blood pressure, as follows: 1) Sympathetic nervous activity is increased after drinking. 2) Ethanol acts directly on the contractility of vascular smooth muscle. Ethanol acutely contracts some arteries and increases their contractile responses to agonists, while it also displays inhibitory effects on vasocontractility in other arteries. Thus, ethanol has two opposite actions, both of which depend on the kinds of vessels and animal species used for the experiments. Intra- and extracellular $\mathrm{Ca}^{2+}$ mobilization and activation of the contractile apparatus have been suggested as mechanisms for ethanol's vasocontractile actions. 3) Chronic alcohol ingestion has been reported to induce a deficiency of blood and intracellular magnesium, which influences cellular $\mathrm{Ca}^{2+}$ homeostasis through attenuation of plasmalemmal ATPase activity. Direct alcohol effects on cardiovascular systems may not be involved in hypertension that develops after long-term habitual drinking. 4) Ethanol affects vascular endothelial functions, inhibiting endothelial NO - and EDHF-dependent vasorelaxations. 5) The serum levels of vasoactive substances such as cathecolamines, renin-aldosterone, prostacyclin, and endothelin have been reported to be affected by alcohol ingestion or ethanol in vitro. 6) In heavy drinkers, alcohol withdrawal results in an elevation of blood pressure due to sympathetic
\end{abstract}


nervous stimulation. 7) Long-term heavy drinking often results in the development of insulin resistance and glucose intolerance, which in turn triggers hypertension. 8) The difference in the genetic polymorphism of acetaldehyde dehydrogenase among Japanese people may not be directly related to development of alcohol-induced hypertension.

As mentioned above, alcohol shows multiple actions on various factors regulating blood pressure. More detailed and integrated mechanisms for alcohol-induced hypertension, which is not a homogeneous disease, remain to be clarified.

Jpn J Hyg 2000; 55: 607-17

\title{
Effect of exercise habits and lifestyles on changes in physical fitness in medical college students: a 3-year follow-up study
}

\author{
Masahisa SAITO \\ Department of Hygiene and Public Health, Osaka Medical College, Osaka
}

\begin{abstract}
This follow-up study investigated the relationship between changes in physical fitness and exercise habits during the 1st and 4th years in healthy medical college students. We also investigated the relation between exercise habits and the lifestyle factors during the same period of time. The subjects were 229 male and 126 female student volunteers who received physical fitness tests and questionnaires (lifestyles and exercise habits) in both the 1st and 4th years.

The results were as follows:

1. A significant association between increment in physical fitness and exercise habits was shown by the fitness score in the 1 st year, and the intensity and frequency of exercise in the 4th year in males. In females, an association was found in the intensity of exercise during the 1 st year, and frequency of exercise during the 4th year. The intensity in 4th year among males (odds ratio(OR): $2.80,95 \%$ confidence interval $(\mathrm{CI}): 1.33-5.87$ ) and the frequency during the 4th year among females (OR: $5.63,95 \% \mathrm{CI}: 2.36-13.43$ ) were associated with a higher odds ratios for improvement in physical fitness than other factors related to exercise habits in 4th year medical students.

2. In the association between exercise habits related to increment in physical fitness and other lifestyles, a significant association was admitted between the intensity of exercise and the frequency of eating breakfast during the 4 th year in males (OR: 2.18, 95\% CI: 1.11-4.28).

These results suggested that the intensity of exercise during the 4 th year in males and the frequency of exercise during the 4th year in females were associated with increments in physical fitness for follow-up. A lifestyle factor related to the intensity in exercise habits has been suggested to be the frequency of eating breakfast (more than 5 days/week) in males.
\end{abstract}

Jpn J Hyg 2001; 55: 618-26 